TRANSACTIONS OF THE

AMERICAN MATHEMATICAL SOCIETY

Volume 355, Number 6, Pages 2205-2222

S 0002-9947(03)03190-8

Article electronically published on February 4, 2003

\title{
ON A MEASURE IN WIENER SPACE AND APPLICATIONS
}

\author{
K. S. RYU AND M. K. IM
}

\begin{abstract}
In this article, we consider a measure in Wiener space, induced by the sum of measures associated with an uncountable set of positive real numbers, and investigate the basic properties of this measure. We apply this measure to the various theories related to Wiener space. In particular, we can obtain a partial answer to Johnson and Skoug's open problems, raised in their 1979 paper. Moreover, we can improve and clarify some theories related to Wiener space.
\end{abstract}

\section{INTRODUCTION}

In 1923, Wiener showed that one can define a reasonable measure on the space $C_{0}[a, b]$ of real-valued continuous functions on a closed interval $[a, b]$ that vanish at $a$, the so-called Wiener space [14]. Since then, the theory of this measure has been investigated extensively and applied to various subjects by many mathematicians and mathematical physicists. So, the various theories related to Wiener space have been widely and deeply developed, and many papers and books related to this space have been published. But in the process of studying Wiener space, one frequently encounters the translation problem, which is one of the difficult problems in the theory of Wiener space. In 1979, Johnson and Skoug raised some open problems concerning translation in Wiener space [8] pp. 164-165].

In this article, we will introduce a measure $\bar{\tau}$ in Wiener space, induced by the sum of measures associated with an uncountable set of positive real numbers, and investigate the basic properties of this measure. Using the concept of $\bar{\tau}$, we will obtain a partial answer to Johnson and Skoug's open problems, raised in their 1979 paper. Furthermore, we can improve and clarify some theories related to Wiener space, for example, the relation between the Lebesgue integral and the Wiener integral, the converse measurability theorem and the theory of the Fourier-Feynman transform.

This article consists of five sections. In the first section, we will introduce some notation, definitions and basic facts which are needed to understand the contents of the next sections. In the second section, we will define a new measure $\bar{\tau}$ in Wiener space and investigate its basic properties. In the third section, we will treat the translation problems for $\bar{\tau}$-measurable sets. Indeed, we will show that if $N$ is

Received by the editors April 6, 2001 and, in revised form, August 29, 2002.

2000 Mathematics Subject Classification. Primary 28C20, 44A15, 46G12, 46T12, 58D20.

Key words and phrases. Wiener measure, scale-invariant measurability, Fourier-Feynman transform.

This work was supported by grant No. 2001-1-10100-011-1 from the Basic Research Program of the Korea Science \& Engineering Foundation. 
$\bar{\tau}$-null, then $N+y$ is $\bar{\tau}$-null for $\bar{\tau}$-a.e. $y$. In the fourth section, we will improve and clarify the converse measurability theorem and the Wiener integration formulas. In the last section, we will apply $\bar{\tau}$-measurability to the theory of Fourier-Feynman transforms, and improve the results in [4], 9].

\section{Preliminaries}

The real number system will be denoted by $\mathbb{R}$. For a natural number $n$, let $\mathbb{R}^{n}$ denote the set of ordered $n$-tuples of real numbers, and let $m_{L}$ denote Lebesgue measure on $\mathbb{R}^{n}$. Let $\mathbb{C}_{+}$be the set of all complex numbers $z$ with $\operatorname{Re} z>0$.

For two real numbers $a$ and $b$ with $a<b$, let $C_{0}[a, b]$ denote Wiener space, that is, the space of all real-valued continuous functions on the closed interval $[a, b]$ that vanish at $a$. Given $x$ in $C_{0}[a, b]$, we define the supremum norm $\|\cdot\|$ given by $\|x\|=\sup _{t \in[a, b]}|x(t)|$. Let $\mathcal{B}$ denote the set of all Borel subsets of $C_{0}[a, b]$ with respect to the supremum norm topology and let $m_{1}$ denote Wiener measure on $\mathcal{B}$. For a positive real number $\lambda$, let $m_{\lambda}$ be the Borel measure on $\mathcal{B}$ given by $m_{\lambda}(B)=m_{1}\left(\lambda^{-1} B\right)$ for $B$ in $\mathcal{B}$. For a positive real number $\lambda$, let $\left(C_{0}[a, b], \overline{\mathcal{B}}_{\lambda}, \bar{m}_{\lambda}\right)$ be the completion of $\left(C_{0}[a, b], \mathcal{B}, m_{\lambda}\right)$. A subset $E$ of $C_{0}[a, b]$ is said to be scaleinvariant measurable provided $E$ belongs to $\overline{\mathcal{B}}_{\lambda}$ for all positive real numbers $\lambda$. Let $\mathcal{S}$ be the set of all scale-invariant measurable subsets. A scale-invariant measurable set $N$ is said to be scale-invariant null provided $\bar{m}_{\lambda}(N)=0$ for all positive real numbers $\lambda$; let $\mathcal{N}$ be the set of all scale-invariant null subsets. A property which holds for all $x$ in $C_{0}[a, b]$ except for a scale-invariant null subset is said to hold scaleinvariant almost everywhere (briefly, s-a.e.). For more detail about scale-invariant measurability and scale change in Wiener space, see [7, pp. 67-78]. If two functions $F$ and $G$ on $C_{0}[a, b]$ are equal s-a.e., we write $F \approx G$.

Given a natural number $n$, let $\sigma_{n}$ be the partition $a=t_{0}<t_{1}<t_{2}<\cdots<$ $t_{2^{n}} \leq b$ where $t_{k}=\frac{k}{2^{n}}(b-a)+a$ for $k=0,1,2, \cdots, 2^{n}$. Given $x$ in $C_{0}[a, b]$, let

$$
S_{\sigma_{n}}(x)=\sum_{k=1}^{2^{n}}\left(x\left(t_{k}\right)-x\left(t_{k-1}\right)\right)^{2} .
$$

For a nonnegative real number $\lambda$, let $C_{\lambda}=\left\{x\right.$ in $C_{0}[a, b] \mid$ the limit $\lim _{n \rightarrow+\infty} S_{\sigma_{n}}(x)$ exists and equals $\left.\lambda^{2}(b-a)\right\}$ and $D=\left\{x\right.$ in $C_{0}[a, b] \mid$ the limit $\lim _{n \rightarrow+\infty} S_{\sigma_{n}}(x)$ does not exist $\}$. Note that $\lambda C_{\mu}=C_{\lambda \mu}$ for all positive real numbers $\lambda$ and $\mu$, and $D$ and the $C_{\lambda}(\lambda \geq 0)$ are all Borel subsets of $C_{0}[a, b]$. Moreover, we know that $C_{0}[a, b]$ is the disjoint union of the family $\left\{C_{\lambda} \mid \lambda \geq 0\right\}$ and $D, m_{\lambda}\left(C_{\lambda}\right)=1$ for any positive real number $\lambda$, and $m_{\lambda}\left(C_{\mu}\right)=0$ for any distinct positive real numbers $\lambda$ and $\mu$ [7], [8].

In [7] and [8] we can find the following theorem.

Theorem 2.1. Let $\lambda$ and $\mu$ be positive real numbers and let $E$ be in $\overline{\mathcal{B}} \sqrt{\lambda^{2}+\mu^{2}}$. Then $E+y$ and $E-y$ are in $\overline{\mathcal{B}}_{\lambda}$ for $\bar{m}_{\mu}$-a.e. $y$, and $\bar{m}_{\lambda}(E+y)$ and $\bar{m}_{\lambda}(E-y)$ are $\bar{m}_{\mu}$-measurable functions of $y$. Similarly, $E+x$ and $E-x$ are in $\overline{\mathcal{B}}_{\mu}$ for $\bar{m}_{\lambda}$-a.e. $x$, 
and $\bar{m}_{\mu}(E+x)$ and $\bar{m}_{\mu}(E-x)$ are $\bar{m}_{\lambda}$-measurable functions of $x$. Furthermore,

$$
\begin{aligned}
& \int_{C_{0}[a, b]} \bar{m}_{\lambda}(E+y) d \bar{m}_{\mu}(y) \\
& \quad=\int_{C_{0}[a, b]} \bar{m}_{\lambda}(E-y) d \bar{m}_{\mu}(y) \\
& =\bar{m}_{\lambda} \times \bar{m}_{\mu}(\{(x, y) \mid x+y \text { is in } E\}) \\
& =\bar{m}_{\sqrt{\lambda^{2}+\mu^{2}}}(E) \\
& =\int_{C_{0}[a, b]} \bar{m}_{\mu}(E+x) d \bar{m}_{\lambda}(x) \\
& =\int_{C_{0}[a, b]} \bar{m}_{\mu}(E-x) d \bar{m}_{\lambda}(x) .
\end{aligned}
$$

Given a number $p$ such that $1 \leq p \leq+\infty, p$ and $p^{\prime}$ will always be related by $\frac{1}{p}+\frac{1}{p^{\prime}}=1$.

Let $F$ be a functional on $C_{0}[a, b]$ such that the integral

$$
J(\lambda)=\int_{C_{0}[a, b]} F\left(\lambda^{-\frac{1}{2}} x\right) d \bar{m}_{1}(x)
$$

exists for almost all positive real $\lambda$. If there exists an analytic function $J^{*}(\lambda)$ in $\mathbb{C}_{+}$ such that $J(\lambda)=J^{*}(\lambda)$ for almost all positive real $\lambda$, then we define this essential analytic extension $J^{*}$ of $J$ to be the analytic Wiener integral of $F$ over $C_{0}[a, b]$ with parameter $\lambda$, and we write

$$
\int_{C_{0}[a, b]}^{\mathrm{anw}_{\lambda}} F(x) d \bar{m}_{1}(x)=J^{*}(\lambda)
$$

for $\lambda$ in $\mathbb{C}_{+}$. For $\lambda$ in $\mathbb{C}_{+}$and for $y$ in $C_{0}[a, b]$, let

$$
\left[T_{\lambda}(F)\right](y) \equiv \int_{C_{0}[a, b]}^{\mathrm{anw}_{\lambda}} F(x+y) d \bar{m}_{1}(x) .
$$

We finish this section by stating a lemma and then using this lemma to prove a theorem. The following lemma follows from [6, Exercise (19.70), p. 339].

Lemma 2.2. Let $I$ be an uncountable set. Let $(\Omega, \mathcal{M})$ be a measurable space and let $\left\{\mu_{\lambda} \mid \lambda\right.$ is in $\left.I\right\}$ be a collection of nonnegative measures on $(\Omega, \mathcal{M})$. For $B$ in $\mathcal{M}$, let

$$
\mu(B)=\sup _{A} \sum_{\lambda \in A} \mu_{\lambda}(B)
$$

where $A$ ranges over all finite subsets of $I$. Then $\mu$ is a measure on $(\Omega, \mathcal{M})$.

Theorem 2.3. Under the assumptions of Lemma 2.2, if $f: \Omega \rightarrow \mathbb{R}$ is nonnegative, bounded, and $\mathcal{M}$-measurable, then

$$
\int_{\Omega} f(x) d \mu(x)=\sup _{A} \sum_{\lambda \in A} \int_{\Omega} f(x) d \mu_{\lambda}(x)
$$

where $A$ ranges over all finite subsets of $I$. 
Proof. For $B$ in $\mathcal{M}$, clearly $\mu(B) \geq \sum_{\lambda \in A} \mu_{\lambda}(B)$ for any finite subset $A$ of $I$. So the inequality

$$
\int_{\Omega} f(x) d \mu(x) \geq \sup _{A} \sum_{\lambda \in A} \int_{\Omega} f(x) d \mu_{\lambda}(x)
$$

holds where $A$ ranges over all finite subsets of $I$. Hence, we must prove that

$$
\int_{\Omega} f(x) d \mu(x) \leq \sup _{A} \sum_{\lambda \in A} \int_{\Omega} f(x) d \mu_{\lambda}(x)
$$

where $A$ ranges over all finite subsets of $I$. Now, let $C$ be in $\mathcal{M}$ with $\mu(C)$ finite. Then there is a sequence $\left\langle\lambda_{i, C}\right\rangle$ in $I$ such that

$$
\begin{gathered}
\mu(C)=\sum_{i=1}^{\infty} \mu_{\lambda_{i, C}}(C) \text { and } \\
\mu_{\lambda}(C)=0 \text { for } \lambda \neq \lambda_{i, C} \quad(i=1,2, \cdots) .
\end{gathered}
$$

Let $K=\left\{\lambda_{i, C} \mid i\right.$ is a natural number $\}$. For $B$ in $\mathcal{M}$, we let

$$
\begin{aligned}
& \mu_{C}^{1}(B)=\sum_{\lambda \in K} \mu_{\lambda}(B) \text { and } \\
& \mu_{C}^{2}(B)=\sup _{A} \sum_{\lambda \in A} \mu_{\lambda}(B)
\end{aligned}
$$

where $A$ ranges over all finite subsets of $I \backslash K$. Then by Lemma $2.2, \mu_{C}^{1}$ and $\mu_{C}^{2}$ are both measures on $(\Omega, \mathcal{M})$ such that $\mu_{C}^{1}+\mu_{C}^{2}=\mu, \mu_{C}^{1}(C)=\mu(C)$ and $\mu_{C}^{2}(C)=0$. Hence, we have

$$
\begin{aligned}
\int_{C} f(x) d \mu(x) & =\int_{C} f(x) d \mu_{C}^{1}(x)+\int_{C} f(x) d \mu_{C}^{2}(x) \\
& =\int_{C} f(x) d \mu_{C}^{1}(x) .
\end{aligned}
$$

Since $f$ is bounded measurable, there is an increasing sequence $\left\langle\varphi_{n}\right\rangle$ of nonnegative simple functions on $C$ such that $\left\langle\varphi_{n}\right\rangle$ converges uniformly to $f$ on $C \mu_{C}^{1}$-a.e. Then we can easily check that

$$
\int_{C} \varphi_{n}(x) d \mu_{C}^{1}(x)=\sum_{i=1}^{\infty} \int_{C} \varphi_{n}(x) d \mu_{\lambda_{i, C}}(x) .
$$

For a natural number $n$, we let $\psi_{n}=\varphi_{n}-\varphi_{n-1}$ where $\varphi_{0}$ is the zero function. Also, for two natural numbers $i$ and $j$, we let

$$
a_{i, j}=\int_{C} \psi_{j}(x) d \mu_{\lambda_{i, C}}(x)
$$

Let $\epsilon$ be any positive real number and let $M \equiv \sup _{\omega \in \Omega}|f(\omega)|$. Since $\mu(C)=\sum_{i=1}^{\infty} \mu_{\lambda_{i, C}}(C)$ 
is finite, there is a natural number $n_{0}$ such that, for $n \geq n_{0}$,

$$
\sum_{i=n+1}^{\infty} \mu_{\lambda_{i, C}}(C)<\frac{\epsilon}{2(M+1)}
$$

and there is a natural number $m_{0}$ such that, for $m \geq m_{0}$,

$$
\left|f(x)-\varphi_{m}(x)\right|<\frac{\epsilon}{2(\mu(C)+1)}
$$

for $\mu_{C^{-}}^{1}$ a.e. $x$. Hence, for $n \geq n_{0}$ and $m \geq m_{0}$,

$$
\begin{aligned}
& \left|\sum_{i=1}^{n} \sum_{j=1}^{m} a_{i, j}-\sum_{i=1}^{\infty} \int_{C} f(x) d \mu_{\lambda_{i, C}}(x)\right| \\
& \quad \leq\left|\sum_{i=1}^{n} \int_{C}\left(\varphi_{m}(x)-f(x)\right) d \mu_{\lambda_{i, C}}(x)\right| \\
& \quad+\left|\sum_{i=n+1}^{\infty} \int_{C} f(x) d \mu_{\lambda_{i, C}}(x)\right| \\
& \quad \leq \frac{\epsilon}{2(\mu(C)+1)} \sum_{i=1}^{n} \mu_{\lambda_{i, C}}(C)+\frac{\epsilon}{2(M+1)} M \\
& \quad<\epsilon .
\end{aligned}
$$

That is, the double series

$$
\left\langle\sum_{i=1}^{n} \sum_{j=1}^{m} a_{i, j}\right\rangle=\left\langle\sum_{i=1}^{n} \int_{C} \varphi_{m}(x) d \mu_{\lambda_{i, C}}(x)\right\rangle
$$

converges absolutely to $\sum_{i=1}^{\infty} \int_{C} f(x) d \mu_{\lambda_{i, C}}(x)$. Thus, we have

$$
\begin{aligned}
& \lim _{m \rightarrow \infty} \lim _{n \rightarrow \infty} \sum_{i=1}^{n} \int_{C} \varphi_{m}(x) d \mu_{\lambda_{i, C}}(x) \\
& =\lim _{n \rightarrow \infty} \lim _{m \rightarrow \infty} \sum_{i=1}^{n} \int_{C} \varphi_{m}(x) d \mu_{\lambda_{i, C}}(x) \\
& =\sum_{i=1}^{\infty} \int_{C} f(x) d \mu_{\lambda_{i, C}}(x) .
\end{aligned}
$$


Thus, we have

$$
\begin{array}{rl}
\int_{C} & f(x) d \mu(x) \\
& \stackrel{(1)}{=} \int_{C} f(x) d \mu_{C}^{1}(x) \\
& \stackrel{(2)}{=} \lim _{m \rightarrow \infty} \int_{C} \varphi_{m}(x) d \mu_{C}^{1}(x) \\
& \stackrel{(3)}{=} \lim _{m \rightarrow \infty} \lim _{n \rightarrow \infty} \sum_{i=1}^{n} \int_{C} \varphi_{m}(x) d \mu_{\lambda_{i, C}}(x) \\
& \stackrel{(4)}{=} \lim _{n \rightarrow \infty} \lim _{m \rightarrow \infty} \sum_{i=1}^{n} \int_{C} \varphi_{m}(x) d \mu_{\lambda_{i, C}}(x) \\
& \stackrel{(5)}{=} \lim _{n \rightarrow \infty} \sum_{i=1}^{n} \int_{C} f(x) d \mu_{\lambda_{i, C}}(x) \\
& \stackrel{(6)}{=} \sum_{i=1}^{\infty} \int_{C} f(x) d \mu_{\lambda_{i, C}}(x) .
\end{array}
$$

Step (1) follows from the equality (2.13). Steps (2) and (5) result from the dominated convergence theorem. By the equality (2.14), we obtain step (3). From the equality (2.17), we have step (4). By the definition of series, we have step (6). Therefore,

$$
\begin{aligned}
& \int_{\Omega} f(x) d \mu(x) \\
& =\sup _{\mu(C)<+\infty} \int_{C} f(x) d \mu(x) \\
& =\sup _{\mu(C)<+\infty} \sum_{i=1}^{\infty} \int_{C} f(x) d \mu_{\lambda_{i, C}}(x) \\
& \leq \sup _{\mu(C)<+\infty} \sum_{i=1}^{\infty} \int_{\Omega} f(x) d \mu_{\lambda_{i, C}}(x) \\
& \leq \sup _{A} \sum_{\lambda \in A} \int_{\Omega} f(x) d \mu_{\lambda}(x)
\end{aligned}
$$

where $A$ ranges over all finite subsets of $I$. Hence, the theorem is proved.

\section{The Measure $\bar{\tau}$ AND its Basic PROperties}

In this section, we will introduce a complete measure $\bar{\tau}$ on the measurable space $\left(C_{0}[a, b], \mathcal{T}\right)$, and we will investigate its basic properties.

Definition 3.1. For $B$ in $\mathcal{B}$, we let

$$
\tau(B)=\sup _{A} \sum_{\lambda \in A} m_{\lambda}(B)
$$

where $A$ ranges over all finite subsets of positive real numbers.

From Lemma 2.2, we know that $\tau$ is a Borel measure on Wiener space. 
Remark 3.2. (1) For $B$ in $\mathcal{B}$ and for a positive real number $\lambda$,

$$
\tau(B)=\tau(\lambda B)
$$

(2) For a Borel subset $B$ of $C_{\lambda}$,

$$
\tau(B)=m_{\lambda}(B) .
$$

(3) $\tau$ is not $\sigma$-finite.

(4) If $E$ is a Borel subset of $\mathbb{R}^{n}$ and $a=t_{0}<t_{1}<t_{2}<\cdots<t_{n} \leq b$, letting $B=\left\{x\right.$ in $C_{0}[a, b] \mid\left(x\left(t_{1}\right), x\left(t_{2}\right), \cdots, x\left(t_{n}\right)\right)$ belongs to $\left.E\right\}$, by the classical Wiener integration formula [7 Theorem 3.3.5, p. 44], $B$ is a Borel subset of $C_{0}[a, b]$ and $\tau(B)$ is either zero or infinite.

(5) Let $f \in L_{2}[a, b]$ and let $P W Z(f)=\left\{x\right.$ in $C_{0}[a, b] \mid$ the Paley-WienerZygmund integral $\int_{a}^{b} f(t) \tilde{d x}(t)$ exists $\}$. Then $\lambda P W Z(f)^{c}$ is a Borel subset of $C_{0}[a, b]$ and $m_{1}\left(\lambda P W Z(f)^{c}\right)=0$ for nonnegative real numbers $\lambda$. So, we have $\bar{\tau}\left(P W Z(f)^{c}\right)=0$.

Definition 3.3. Let $\left(C_{0}[a, b], \mathcal{T}, \bar{\tau}\right)$ be the completion of the measure space $\left(C_{0}[a, b]\right.$, $\mathcal{B}, \tau)$. A property that holds for all $x$ in $C_{0}[a, b]$ except for a $\bar{\tau}$-null set is said to hold $\bar{\tau}$-almost everywhere (briefly, $\bar{\tau}$-a.e.). If two functionals $F$ and $G$ on $C_{0}[a, b]$ are equal $\bar{\tau}$-a.e., we write $F \stackrel{\bar{\tau}}{\approx} G$.

Remark 3.4. (1) The relation $\underset{\mathcal{\tau}}{\approx}$ is an equivalence relation on the class of all $\bar{\tau}$ measurable Wiener functionals.

(2) $\bar{\tau}$ is not $\sigma$-finite.

(3) If $N$ is $\bar{\tau}$-null, then $N$ is in $\mathcal{N}$.

(4) The set $\mathcal{T}$ is a subset of $\mathcal{S}$.

(5) For $E$ in $\mathcal{S}$, let $\mu(E)=\sup _{A} \sum_{\lambda \in A} \bar{m}_{\lambda}(E)$ where $A$ ranges over all finite subsets of positive real numbers. Then by Lemma $2.2, \mu$ is a measure on $\left(C_{0}[a, b], \mathcal{S}\right)$ and the measure space $\left(C_{0}[a, b], \mathcal{S}, \mu\right)$ is a complete measure space.

Now we will show the relationships between the $\sigma$-algebras $\mathcal{B}, \mathcal{T}, \mathcal{S}$ and $\overline{\mathcal{B}}_{\lambda}$ where $\lambda$ is a positive real number.

The next lemma follows from [3], 7], [8].

Lemma 3.5. For an arbitrary function $f:(0,+\infty) \rightarrow[0,1]$, there is a set $E$ in $\mathcal{S}$ such that $f(\lambda)=\bar{m}_{\lambda}(E)$ for positive real numbers $\lambda$.

The following lemma was established in [13].

Lemma 3.6. For $B$ in $\mathcal{B}, m_{\lambda}(B)$ is a Borel measurable function of $\lambda$.

By Definition 3.3, for $E$ in $\mathcal{T}$, we can write $E=B \cup N$ such that $B$ is in $\mathcal{B}$, $N$ is $\bar{\tau}$-null and $B \cap N=\emptyset$. Then, by Lemma 3.6, $\bar{m}_{\lambda}(E)=m_{\lambda}(B)$ is a Borel measurable function of $\lambda$. Hence, we have the following.

Corollary 3.7. For $E$ in $\mathcal{T}, \bar{m}_{\lambda}(E)$ is a Borel measurable function of $\lambda$.

Theorem 3.8. $\mathcal{B} \underset{\neq}{\subset} \underset{F}{\subset \mathcal{S}} \underset{\neq}{\subset} \overline{\mathcal{B}}_{\lambda}$ holds for all positive real numbers $\lambda$.

Proof. From [7] and [8] one can find that $\mathcal{B} \subsetneq \mathcal{S} \subseteq \overline{\mathcal{B}}_{\lambda}$ is true for all positive real numbers $\lambda$. Since it is trivial that $\mathcal{B} \subset \mathcal{T} \subset \mathcal{S}$, it remains to show that $\mathcal{B} \neq \mathcal{T}$ and $\mathcal{T} \neq \mathcal{S}$. Let $t$ be fixed with $a<t \leq b$ and let $P: C_{0}[a, b] \rightarrow \mathbb{R}$ be a function with 
$P(x)=x(t)$. Let $N$ be a Lebesgue null subset of $\mathbb{R}$ but not a Borel measurable subset. Then there is a Borel measurable subset $N^{*}$ of $\mathbb{R}$ such that $N \subset N^{*}$ and $m_{L}\left(N^{*}\right)=0$. For any positive real number $\lambda, \lambda^{-1} N^{*}$ is a Borel measurable subset with $m_{L}\left(\lambda^{-1} N^{*}\right)=0$. Hence, by the classical Wiener integration formula, we know that $m_{\lambda}\left(P^{-1}\left(N^{*}\right)\right)=m_{1}\left(\lambda^{-1} P^{-1}\left(N^{*}\right)\right)=m_{1}\left(P^{-1}\left(\lambda^{-1} N^{*}\right)\right)=0$; that is, $P^{-1}\left(N^{*}\right)$ is $\tau$-null. Since $P^{-1}(N) \subset P^{-1}\left(N^{*}\right), P^{-1}(N)$ is in $\mathcal{T}$. But, by the converse measurability theorem [5], [7], we know that $P^{-1}(N)$ is not in $\mathcal{B}$, which implies that $\mathcal{T} \neq \mathcal{B}$. Now, if $K$ is a non-measurable subset of $(0,+\infty)$, letting $F=\bigcup_{\lambda \in K} C_{\lambda}, \bar{m}_{\lambda}(F)$ is not a measurable function of $\lambda$. So by Corollary 2.7, $F$ is not in $\mathcal{T}$. Trivially, $F$ is in $\mathcal{S}$, which implies that $\mathcal{T} \neq \mathcal{S}$, as desired.

Remark 3.9 (The generalized Radon-Nikodým theorem [10, 11]). Let $I$ be an arbitrary subset of positive real numbers. For $B$ in $\mathcal{B}$, let

$$
\tau_{I}(B)=\sup _{A} \sum_{\lambda \in A} m_{\lambda}(B)
$$

where $A$ ranges over all finite subsets of $I$. Then by Lemma 1.2, $\tau_{I}$ is a measure on $\left(C_{0}[a, b], \mathcal{B}\right)$ and $\tau_{I}$ is absolutely continuous with respect to $\tau$. Moreover, for $B$ in $\mathcal{B}$ with $\tau(B)$ finite,

$$
\tau_{I}(B)=\int_{B} \chi_{M}(x) d \tau(x)
$$

where $M=\bigcup_{\lambda \in I} C_{\lambda}$ and $\chi_{M}$ is a characteristic function associated with a set $M$. Here, $\chi_{M}$ may be not measurable, but for a set $B$ in $\mathcal{B}$ with $\tau(B)$ finite, the restriction of $\chi_{M}$ to $B$ is measurable. Similarly, equation (3.5) also holds with $\tau$ and $\tau_{I}$ replaced by $\bar{\tau}$ and $\bar{\tau}_{I}$, respectively [11].

For $E$ in $\mathcal{T}$ with $\bar{\tau}(E)$ positive, there is a positive real number $\lambda_{0}$ such that $\bar{m}_{\lambda_{0}}(E)$ is positive. Letting $F=E \cap C_{\lambda_{0}}, F$ is in $\mathcal{T}$ with $F \subset E$ and $0<$ $\bar{\tau}(F)=\bar{m}_{\lambda_{0}}(E) \leq 1$; that is, $\bar{\tau}$ has the finite subset property [11, p. 68]. Let $\mathcal{R}=\{A \times B \mid A, B$ are in $\mathcal{T}\}$. For $A \times B$ in $\mathcal{R}$, we let $\alpha(A \times B)=\bar{\tau}(A) \bar{\tau}(B)$. Then $\alpha$ is countably additive on a semi-algebra $\mathcal{R}$. Since $\bar{\tau}$ has the finite subset property, by [11, Exercise 6, p. 323], there is an unique extension $\bar{\tau} \times \bar{\tau}$ of $\alpha$ on the smallest $\sigma$-algebra $\mathcal{T} \otimes \mathcal{T}$ containing $\mathcal{R}$. Here we will establish the integration formula for the product measure $\bar{\tau} \times \bar{\tau}$, which plays a very important role in this article.

Theorem 3.10. Let $f: C_{0}[a, b] \times C_{0}[a, b] \rightarrow \mathbb{R}$ be nonnegative $\bar{\tau} \times \bar{\tau}$-measurable. Then

$$
\begin{aligned}
& \int_{C_{0}[a, b] \times C_{0}[a, b]} f(x, y) d \bar{\tau} \times \bar{\tau}(x, y) \\
& \quad=\sup _{A} \sup _{B} \sum_{\lambda \in A} \sum_{\mu \in B} \int_{C_{0}[a, b] \times C_{0}[a, b]} f(x, y) d \bar{m}_{\lambda} \times \bar{m}_{\mu}(x, y)
\end{aligned}
$$

where $A$ and $B$ range over all finite subsets of positive real numbers.

Proof. Trivially, we have $\bar{\tau} \times \bar{\tau}(E) \geq \sum_{\lambda \in A} \sum_{\mu \in B} \bar{m}_{\lambda} \times \bar{m}_{\mu}(E)$ for all $\bar{\tau} \times \bar{\tau}$-measurable subsets $E$ and for any finite subsets $A$ and $B$ of positive real numbers. Hence, we 
have

$$
\begin{aligned}
& \int_{C_{0}[a, b] \times C_{0}[a, b]} f(x, y) d \bar{\tau} \times \bar{\tau}(x, y) \\
& \quad \geq \sup _{A} \sup _{B} \sum_{\lambda \in A} \sum_{\mu \in B} \int_{C_{0}[a, b] \times C_{0}[a, b]} f(x, y) d \bar{m}_{\lambda} \times \bar{m}_{\mu}(x, y) .
\end{aligned}
$$

If

$$
\sup _{A} \sup _{B} \sum_{\lambda \in A} \sum_{\mu \in B} \int_{C_{0}[a, b] \times C_{0}[a, b]} f(x, y) d \bar{m}_{\lambda} \times \bar{m}_{\mu}(x, y)=+\infty
$$

then (3.6) clearly holds. Now, we suppose that

$$
\sup _{A} \sup _{B} \sum_{\lambda \in A} \sum_{\lambda \in B} \int_{C_{0}[a, b] \times C_{0}[a, b]} f(x, y) d \bar{m}_{\lambda} \times \bar{m}_{\mu}(x, y)
$$

is finite. Letting

$$
T=\left\{(\lambda, \mu) \mid \int_{C_{0}[a, b] \times C_{0}[a, b]} f(x, y) d \bar{m}_{\lambda} \times \bar{m}_{\mu}(x, y) \text { is positive }\right\}
$$

we see that $T$ is countable, say $T=\left\{\left(\lambda_{i}, \mu_{i}\right) \mid i\right.$ is a natural number $\}$. Let $L=$ $\bigcup_{i=1}^{\infty} C_{\lambda_{i}} \times C_{\mu_{i}}$ and let $M=C_{0}[a, b] \times C_{0}[a, b] \backslash L$. Then $L$ and $M$ are all Borel subsets, and $L \cap M=\emptyset$. Hence, we have

$$
\begin{aligned}
& \int_{C_{0}[a, b] \times C_{0}[a, b]} f(x, y) d \bar{\tau} \times \bar{\tau}(x, y) \\
& \quad=\int_{L} f(x, y) d \bar{\tau} \times \bar{\tau}(x, y)+\int_{M} f(x, y) d \bar{\tau} \times \bar{\tau}(x, y) \\
& \quad=\sum_{i=1}^{\infty} \int_{C_{\lambda_{i}} \times C_{\mu_{i}}} f(x, y) d \bar{m}_{\lambda_{i}} \times \bar{m}_{\mu_{i}}(x, y)+\int_{M} f(x, y) d \bar{\tau} \times \bar{\tau}(x, y)
\end{aligned}
$$

If we prove that $\int_{M} f(x, y) d \bar{\tau} \times \bar{\tau}(x, y)=0$, then equality (3.6) holds. Let $B$ be a $\bar{\tau} \times \bar{\tau}$-measurable subset of $M$ with $\bar{\tau} \times \bar{\tau}(B)$ finite. Then there is a disjoint sequence $\left\langle G_{n}\right\rangle$ in $\mathcal{R}$ such that $B \subset \bigcup_{n=1}^{\infty} G_{n} \subset M$ and $\sum_{n=1}^{\infty} \bar{\tau} \times \bar{\tau}\left(G_{n}\right)<\bar{\tau} \times \bar{\tau}(B)+1$. Put $G_{n}=E_{n} \times F_{n}$ for each natural number $n$. Then there is a subsequence $\left\langle G_{n_{i}}\right\rangle$ of $\left\langle G_{n}\right\rangle$ such that $\bar{\tau}\left(E_{n_{i}}\right)$ and $\bar{\tau}\left(F_{n_{i}}\right)$ are all finite for all natural numbers $i$ and $\bar{\tau} \times \bar{\tau}\left(B \backslash \bigcup_{n=1}^{\infty} G_{n_{i}}\right)=0$. Then for each natural number $n_{i}$, there are two sequences $\left\langle\lambda_{n_{i, j}}\right\rangle$ and $\left\langle\mu_{n_{i, j}}\right\rangle$ such that $\bar{\tau}\left(E_{n_{i}}\right)=\sum_{j=1}^{\infty} \bar{m}_{\lambda_{n_{i, j}}}\left(E_{n_{i}}\right)$ and $\bar{\tau}\left(F_{n_{i}}\right)=\sum_{j=1}^{\infty} \bar{m}_{\mu_{n_{i, j}}}\left(F_{n_{i}}\right)$. 
Then

$$
\begin{array}{rl}
\int_{B} & f(x, y) d \bar{\tau} \times \bar{\tau}(x, y) \\
= & \int_{B \cap\left(\cup_{i=1}^{\infty} E_{n_{i}} \times F_{n_{i}}\right)} f(x, y) d \bar{\tau} \times \bar{\tau}(x, y) \\
& +\int_{B \backslash\left(\cup_{i=1}^{\infty} E_{n_{i}} \times F_{n_{i}}\right)} f(x, y) d \bar{\tau} \times \bar{\tau}(x, y) \\
\leq & \sum_{i=1}^{\infty} \int_{E_{n_{i}} \times F_{n_{i}}} f(x, y) d \bar{\tau} \times \bar{\tau}(x, y) \\
\leq & \sum_{i=1}^{\infty} \sum_{j=1}^{\infty} \sum_{k=1}^{\infty} \int_{C_{\lambda_{n_{i, j}}} \times C_{\mu_{n_{i, k}}}} f(x, y) d \bar{m}_{\lambda_{n_{i, j}}} \times \bar{m}_{\mu_{n_{i, k}}}(x, y) \\
& \left(\lambda_{n_{i, j}}, \mu_{n_{i, k}}\right) \notin T \\
= & 0 .
\end{array}
$$

Hence, $\int_{M} f(x, y) d \bar{\tau} \times \bar{\tau}(x, y)=\sup _{D} \int_{D} f(x, y) d \bar{\tau} \times \bar{\tau}(x, y)=0$, as desired where $D$ ranges over all measurable subsets of $M$ with $\bar{\tau} \times \bar{\tau}(D)$ finite.

Example 3.11. In general, Theorem 3.10 does not hold. Let $(\mathbb{R}, \mathcal{M})$ be a Lebesgue measurable space. For a positive real number $\lambda$, let $\mu_{\lambda}$ be the Dirac measure on $(\mathbb{R}, \mathcal{M})$. For a positive real number $\lambda$ with $\lambda \neq 1$, let $\nu_{\lambda}$ be the zero measure on $(\mathbb{R}, \mathcal{M})$, and let $\nu_{1}$ be the usual Lebesgue measure on $(\mathbb{R}, \mathcal{M})$. For $E$ in $\mathcal{M}$, let

$$
\begin{gathered}
\mu(E)=\sup _{A} \sum_{\lambda \in A} \mu_{\lambda}(E) \quad \text { and } \\
\nu(E)=\sup _{A} \sum_{\lambda \in A} \nu_{\lambda}(E)
\end{gathered}
$$

where $A$ ranges over all finite subsets of $\mathbb{R}$. Then $\mu$ is the counting measure on $(\mathbb{R}, \mathcal{M})$ and $\nu$ is the Lebesgue measure on $(\mathbb{R}, \mathcal{M})$. Let $\Delta=\{(x, x) \mid x$ is in $\mathbb{R}\}$. Then $\Delta$ is measurable in the product space $\mathbb{R} \times \mathbb{R}$, and

$$
\int_{\mathbb{R} \times \mathbb{R}} \int_{\Delta}(x, y) d \mu \times \nu(x, y)=+\infty
$$

but

$$
\sup _{A} \sup _{B} \sum_{t \in A} \sum_{s \in B} \int_{\mathbb{R}} \int_{\mathbb{R}} \chi_{\Delta}(x, y) d \nu_{s}(y) d \mu_{t}(x)=0
$$

where $A$ and $B$ range over all finite subsets of $\mathbb{R}$.

\section{Translation in Wiener SPace}

It is a well-known fact that if $X$ is an infinite-dimensional vector space, there is no nonzero quasi-invariant measure $\mu$ on $\left(X, \mathcal{B}_{R}\right)$ where $R$ is a linear subspace of the dual space of $X$ and $\mathcal{B}_{R}$ is the smallest $\sigma$-algebra on $X$ in which every $f$ in $R$ is measurable; that is, there is no nonzero measure $\mu$ such that $\mu(N)=0$ implies $\mu(N+y)=0$ for all $y$ in $X$ [15, Corollary 1, p. 142]. Since the Wiener space $C_{0}[a, b]$ with the supremum norm is a real separable infinite-dimensional Banach 
space, there is no nonzero quasi-invariant measure on Wiener space $C_{0}[a, b]$. It is natural to wonder how many $y$ in $C_{0}[a, b]$ exist such that $\bar{\tau}(N)=0$ but $\bar{\tau}(N+y) \neq 0$.

Actually, Cameron showed in [2] that there is an $\bar{m}_{1}$-measurable set $E$ such that $E+y$ is not $\bar{m}_{1}$-measurable for $\bar{m}_{1}$-a.e. $y$, and Johnson and Skoug, in their 1979 paper [8], raised the following open problems.

(1) Let $E$ be in $\mathcal{S}$. Is it true that $E+y$ is in $\mathcal{S}$ for s-a.e. $y$ ?

(2) Let $N$ be in $\mathcal{N}$. Is $N+y$ in $\mathcal{N}$ true for s-a.e. $y$ ?

Theorem 4.1. Let $N$ be $\bar{\tau}$-null. Then $N+y$ and $N-y$ are $\bar{\tau}$-null for $\bar{\tau}$-a.e. $y$.

Proof. Let $N$ be $\bar{\tau}$-null. Consider a function $\Phi: C_{0}[a, b] \times C_{0}[a, b] \rightarrow C_{0}[a, b]$ such that $\Phi(x, y)=x-y$. Since $N$ is $\bar{\tau}$-null, there is a Borel set $N^{*}$ in $C_{0}[a, b]$ such that $N \subset N^{*}$ and $\tau\left(N^{*}\right)=0$. Because $\Phi$ is continuous, $\Phi^{-1}\left(N^{*}\right)$ is also a Borel subset of $C_{0}[a, b] \times C_{0}[a, b]$. Hence, by Theorem 2.1 and Theorem 3.10,

$$
\begin{aligned}
& \int_{C_{0}[a, b] \times C_{0}[a, b]} \chi_{\Phi^{-1}\left(N^{*}\right)}(x, y) d \bar{\tau} \times \bar{\tau}(x, y) \\
& =\sup _{A} \sup _{B} \sum_{\lambda \in A} \sum_{\mu \in B} \int_{C_{0}[a, b] \times C_{0}[a, b]} \chi_{\Phi^{-1}\left(N^{*}\right)}(x, y) d \bar{m}_{\lambda} \times \bar{m}_{\mu}(x, y) \\
& =\sup _{A} \sup _{B} \sum_{\lambda \in A} \sum_{\mu \in B} \int_{C_{0}[a, b] \times C_{0}[a, b]} \chi_{N^{*}+y}(x) d \bar{m}_{\lambda} \times \bar{m}_{\mu}(x, y) \\
& =\sup _{A} \sup _{B} \sum_{\lambda \in A} \sum_{\lambda \in B} \int_{C_{0}[a, b]} \chi_{N^{*}}(z) d \bar{m} \sqrt{\lambda^{2}+\mu^{2}}(z) \\
& =0
\end{aligned}
$$

where $A$ and $B$ range over all finite subsets of positive real numbers. Hence, $\bar{\tau} \times$ $\bar{\tau}\left(\Phi^{-1}\left(N^{*}\right)\right)=0$. By [12, Lemma 17, p. 306] for $\bar{\tau}$-a.e. $y$,

$$
\bar{\tau}\left(\left[\Phi^{-1}\left(N^{*}\right)\right]_{y}\right)=\bar{\tau}\left(N^{*}+y\right)=0
$$

where $[A]_{y}$ means the $y$ cross section of a set $A$. Since $N \subset N^{*}$, we have that $\bar{\tau}(N+y)=0$ for $\bar{\tau}$-a.e. $y$. Similarly, it follows that $N-y$ is $\bar{\tau}$-null for $\bar{\tau}$-a.e. $y$, which concludes the proof of Theorem 4.1.

Theorem 4.2. If $E$ is in $\mathcal{T}$, then $E+y$ and $E-y$ are in $\mathcal{T}$ for $\bar{\tau}$-a.e. $y$.

Proof. Let $E$ be in $\mathcal{T}$. Then there are two sets $B$ and $N$ such that $B$ is in $\mathcal{B}, N$ is $\bar{\tau}$-null, $B \cap N=\emptyset$ and $E=B \cup N$. Trivially, $B+y$ and $B-y$ are in $\mathcal{B}$ for all $y$ in $C_{0}[a, b]$, and by Theorem $4.1, N+y$ and $N-y$ are $\bar{\tau}$-null for $\bar{\tau}$-a.e. $y$. Hence $E+y=(B+y) \cup(N+y)$ and $E-y=(B-y) \cup(N-y)$ are in $\mathcal{T}$ for $\bar{\tau}$-a.e. $y$, as desired.

Theorem 4.3. If $f$ is a $\mathcal{T}$-measurable function, then, for $\bar{\tau}$-a.e. $y, f(x+y)$ and $f(x-y)$ are $\mathcal{T}$-measurable functions of $x$.

Proof. Let $f$ be $\mathcal{T}$-measurable. Then there is a sequence $\left\langle\varphi_{n}\right\rangle$ of $\mathcal{T}$-simple functions with $\bar{\tau}\left(K_{1}^{c}\right)=0$ where $K_{1} \equiv\left\{x\right.$ in $C_{0}[a, b] \mid$ the limit $\lim _{n \rightarrow \infty} \varphi_{n}(x)$ exists and equals $f(x)\}$. Let $\Phi: C_{0}[a, b] \times C_{0}[a, b] \rightarrow C_{0}[a, b]$ be a function with $\Phi(x, y)=x-y$. By a method similar to that used in the proof of Theorem 4.1, we can show that $\Phi^{-1}\left(K_{1}^{c}\right)$ is $\bar{\tau} \times \bar{\tau}$-null, and so for $\bar{\tau}$-a.e. $y,\left[\Phi^{-1}\left(K_{1}^{c}\right)\right]_{y}$ is $\bar{\tau}$-null. Since if $\varphi_{n}$ is $\mathcal{T}$-simple then there is a $\bar{\tau}$-measurable subset $K_{2}$ of $C_{0}[a, b]$ such that $\bar{\tau}\left(K_{2}\right)=0$ and $\varphi_{n}(x+y)$ is 
also $\mathcal{T}$-simple for $y$ in $K_{2}$ and $\left[\Phi^{-1}\left(K_{1}^{c}\right)\right]_{y}=\left\{x\right.$ in $C_{0}[a, b] \mid$ the limit $\lim _{n \rightarrow \infty} \varphi_{n}(x+y)$ exists and equals $f(x+y)\}^{c}$, it follows that $f(x+y)$ is a $\mathcal{T}$-measurable function of $x$ for $y$ in $K_{1} \cap K_{2}^{c}$, and $\bar{\tau}\left(K_{1}^{c} \cup K_{2}\right)=0$. By a method similar to that used in the proof of this theorem, it is not hard to prove that for $\bar{\tau}$-a.e. $y, f(x-y)$ is a $\bar{\tau}$-measurable function of $x$, which concludes the proof of Theorem 4.3.

Theorem 4.4. Let $f: C_{0}[a, b] \rightarrow \mathbb{R}$ be nonnegative and $\bar{\tau}$-measurable. Then $\int_{C_{0}[a, b] \times C_{0}[a, b]} f(x+y) d \bar{\tau} \times \bar{\tau}(x, y)$ is either zero or infinite.

Proof. From Theorem 2.1 and Theorem 3.10, we have

$$
\begin{aligned}
& \int_{C_{0}[a, b] \times C_{0}[a, b]} f(x+y) d \bar{\tau} \times \bar{\tau}(x, y) \\
& =\sup _{A} \sup _{B} \sum_{\lambda \in A} \sum_{\mu \in B} \int_{C_{0}[a, b] \times C_{0}[a, b]} f(x+y) d \bar{m}_{\lambda} \times \bar{m}_{\mu}(x, y) \\
& =\sup _{A} \sup _{B} \sum_{\lambda \in A} \sum_{\mu \in B} \int_{C_{0}[a, b]} f(z) d \bar{m} \sqrt{\lambda^{2}+\mu^{2}}(z)
\end{aligned}
$$

where $A$ and $B$ range over all finite subsets of positive real numbers.

If $\int_{C_{0}[a, b]} f(z) d \bar{m} \sqrt{\lambda_{0}^{2}+\mu_{0}^{2}}(z)$ is positive for some positive real numbers $\lambda_{0}$ and $\mu_{0}$, then for all $(\lambda, \mu)$ in $K=\left\{(\lambda, \mu) \mid \sqrt{\lambda^{2}+\mu^{2}}=\sqrt{\lambda_{0}^{2}+\mu_{0}^{2}}, \lambda>0\right.$ and $\left.\mu>0\right\}$ the integral $\int_{C_{0}[a, b]} f(z) d \bar{m} \sqrt{\lambda^{2}+\mu^{2}}(z)$ is positive and $K$ is uncountable; so

$$
\int_{C_{0}[a, b] \times C_{0}[a, b]} f(x+y) d \bar{\tau} \times \bar{\tau}(x, y)
$$

is infinite.

\section{THE CONVERSE MEASURABILITY THEOREM AND THE WIENER INTEGRATION FORMULA}

In this section, we will treat the converse measurability theorem and the Wiener integration formula in the light of $\bar{\tau}$-measurability.

Throughout this section, we adopt the following notation: $\leq b$.

(1) Let $\vec{t}=\left(t_{1}, t_{2}, \cdots, t_{n}\right)$ be a vector in $\mathbb{R}^{n}$ with $a=t_{0}<t_{1}<t_{2}<\cdots<t_{n}$

(2) Let $P_{\vec{t}}: C_{0}[a, b] \rightarrow \mathbb{R}^{n}$ be a function with $P_{\vec{t}}(x)=\left(x\left(t_{1}\right), x\left(t_{2}\right), \cdots, x\left(t_{n}\right)\right)$.

(3) For a positive real number $\lambda$, let

$$
W_{n}(\lambda, \vec{t}, \vec{u})=\left[(2 \pi \lambda)^{n} \prod_{i=1}^{n}\left(t_{i}-t_{i-1}\right)\right]^{-\frac{1}{2}} \exp \left\{-\frac{1}{2 \lambda} \sum_{i=1}^{n} \frac{\left(u_{i}-u_{i-1}\right)^{2}}{t_{i}-t_{i-1}}\right\} .
$$

Theorem 5.1 (The converse measurability theorem). Let $E$ be a subset of $\mathbb{R}^{n}$. The following statements are equivalent:

(1) $E$ is Lebesgue measurable.

(2) $P_{\vec{t}}^{-1}(E)$ is $\bar{\tau}$-measurable.

(3) For any positive real number $\lambda, P_{\vec{t}}^{-1}(E)$ is $\bar{m}_{\lambda}$-measurable, that is, $P_{\vec{t}}^{-1}(E)$ is in $\mathcal{S}$.

Proof. Let $E$ be a Lebesgue measurable set in $\mathbb{R}^{n}$. Then we can write $E=B \cup N$ where $B$ is a Borel measurable set in $\mathbb{R}^{n}, N$ is a Lebesgue null set and $B \cap N=\emptyset$. 
Since $P_{\vec{t}}$ is continuous, $P_{\vec{t}}^{-1}(B)$ is Borel measurable. Also, there is a Borel null subset $N^{*}$ of $\mathbb{R}^{n}$ such that $N \subset N^{*}$. Then, for any positive real number $\lambda$,

$$
\begin{aligned}
& m_{\lambda}\left(P_{\vec{t}}^{-1}\left(N^{*}\right)\right)=m_{1}\left(\lambda^{-1} P_{\vec{t}}^{-1}\left(N^{*}\right)\right)=m_{1}\left(P_{\vec{t}}^{-1}\left(\lambda^{-1} N^{*}\right)\right) \\
& \quad=\int_{\mathbb{R}^{n}} \chi_{\lambda^{-1} N^{*}}\left(u_{1}, u_{2}, \cdots, u_{n}\right) W_{n}(1, \vec{t}, \vec{u}) d m_{L}(\vec{u}) \\
& \quad=0
\end{aligned}
$$

which implies that $P_{\vec{t}}^{-1}\left(N^{*}\right)$ is $\tau$-null. Since $P_{\vec{t}}^{-1}(N) \subset P_{\vec{t}}^{-1}\left(N^{*}\right)$ and $P_{\vec{t}}^{-1}(E)=$ $P_{\vec{t}}^{-1}(B) \cup P_{\vec{t}}^{-1}(N), P_{\vec{t}}^{-1}(E)$ is $\bar{\tau}$-measurable. Hence (1) implies (2). It is clear that (2) implies (3). By the classical converse measurability theorem [5], we know that (3) implies (1).

Theorem 5.2 (The Wiener integration formula). Let $f: \mathbb{R}^{n} \rightarrow \mathbb{R}$ be a function. Then the following statements are equivalent:

(1) $f$ is Lebesgue measurable.

(2) $f \circ P_{\vec{t}}$ is $\bar{\tau}$-measurable.

(3) $f \circ P_{\vec{t}}$ is $\mathcal{S}$-measurable.

Furthermore, if any one of the conditions (1), (2), or (3) holds, then for any positive real number $\lambda$,

$$
\begin{aligned}
& \int_{C_{0}[a, b]} f \circ P_{\vec{t}}(x) d \bar{m}_{1}(x)=\int_{C_{\lambda}} f \circ P_{\vec{t}}(\lambda x) d \bar{m}_{\lambda}(x) \\
& \quad=\int_{\mathbb{R}^{n}} f(u) W_{n}(1, \vec{t}, \vec{u}) d m_{L}(\vec{u}) \\
& =\int_{\mathbb{R}^{n}} f\left(\lambda^{-\frac{1}{2}} \vec{u}\right) W_{n}(\lambda, \vec{t}, \vec{u}) d m_{L}(\vec{u})
\end{aligned}
$$

where if any one of the four integrals in equation (5.2) is defined, whether finite or infinite, then each of the other three integrals is also defined and equality holds.

Proof. Let $E$ be a Lebesgue measurable set in $\mathbb{R}^{n}$ and let $f(\vec{u})=\chi_{E}(\vec{u})$. Then we can write $E=B \cup N$ where $B$ is a Borel subset of $\mathbb{R}^{n}, N$ is a Lebesgue null set and $B \cap N=\emptyset$. By the proof of Theorem 5.1, $P_{\vec{t}}^{-1}(N)$ is $\bar{\tau}$-null. Since $P_{\vec{t}}^{-1}(B)$ is Borel, trivially, $f \circ P_{\vec{t}}(x)=\chi_{P_{\vec{t}}^{-1}(B)}(x)+\chi_{P_{\vec{t}}^{-1}(N)}(x)$ is $\bar{\tau}$-measurable. We assume that $f$ is simple, say $f=\sum_{i=1}^{n} a_{i} \chi_{E_{i}}$ where the $a_{i}(i=1,2, \cdots, n)$ are real numbers and the $E_{i} \quad(i=1,2, \cdots, n)$ are pairwise disjoint Lebesgue measurable sets in $\mathbb{R}^{n}$. Then clearly $f \circ P_{\vec{t}}(x)=\sum_{i=1}^{n} a_{i} \chi_{P_{\vec{t}}^{-1}\left(E_{i}\right)}(x)$ is $\bar{\tau}$-measurable. Now, let $f$ be an arbitrary measurable function on $\mathbb{R}^{n}$. Then there is a sequence $\left\langle\varphi_{n}\right\rangle$ of simple functions such that $\left\langle\varphi_{n}\right\rangle$ converges to $f, m_{L}$-a.e. Let $K \equiv\left\{\vec{u}\right.$ in $\mathbb{R}^{n} \mid$ the limit $\lim _{n \rightarrow \infty} \varphi_{n}(\vec{u})$ exists and $\left.\lim _{n \rightarrow \infty} \varphi_{n}(\vec{u})=f(\vec{u})\right\}$. Then $K^{c}$ is Lebesgue null and $\left\{x\right.$ in $C_{0}[a, b] \mid$ the limit $\lim _{n \rightarrow \infty} \varphi_{n} \circ P_{\vec{t}}(x)$ exists and $\left.\lim _{n \rightarrow \infty} \varphi_{n} \circ P_{\vec{t}}(x)=f \circ P_{\vec{t}}(x)\right\}=P_{\vec{t}}^{-1}\left(K^{c}\right)$. By the proof of Theorem 5.1, $P_{\vec{t}}^{-1}\left(K^{c}\right)$ is $\bar{\tau}$-null. So for all natural numbers $n, \varphi_{n} \circ P_{\vec{t}}$ is $\bar{\tau}$-measurable and $P_{\vec{t}}^{-1}(K)$ is $\bar{\tau}$-null. Hence $f \circ P_{\vec{t}}$ is $\bar{\tau}$-measurable. So the proof of "(1) implies (2)" is finished. It is trivial that (2) implies (3) by Theorem 3.8. To prove that (3) implies (1), we assume that $f \circ P_{\vec{t}}$ is $\mathcal{S}$-measurable. Then, for any 
Borel subset $B$ of $\mathbb{R},\left(f \circ P_{\vec{t}}\right)^{-1}(B)=P_{\vec{t}}^{-1}\left(f^{-1}(B)\right)$ is $\bar{m}_{1}$-measurable. Hence, by the converse measurability theorem [5], $f^{-1}(B)$ is Lebesgue measurable, as desired. By the classical Wiener integration formula [7] and the change of variables, we can show that the equalities in (5.2) hold.

Remark 5.3. Let $\mathcal{M}(\bar{\tau})$ be the set of all $\bar{\tau}$-measurable functions $F$ of the form $F=g \circ P_{\vec{t}}$ on $C_{0}[a, b]$ where $g$ is a complex-valued function on $\mathbb{R}^{n}$, and let $\mathcal{M}\left(m_{L}\right)$ be the set of all $m_{L}$-measurable functions on $\mathbb{R}^{n}$. For $F$ in $\mathcal{M}(\bar{\tau})$ let

$$
[F]_{\bar{\tau}}=\{G \text { in } \mathcal{M}(\bar{\tau}) \mid G \stackrel{\bar{\tau}}{\approx} F\}
$$

and for $f$ in $\mathcal{M}\left(m_{L}\right)$ let

$$
[f]_{m_{L}}=\left\{g \text { in } \mathcal{M}\left(m_{L}\right) \mid g=f m_{L^{-} \text {-a.e. }}\right\} \text {. }
$$

Let $T: \mathcal{M}\left(m_{L}\right) \rightarrow \mathcal{M}(\bar{\tau})$ be a function with $T(f)=f \circ P_{\vec{t}}$. By the elementary theory of measure, one can check easily that the following statements hold.

(1) $T$ is bijective.

(2) If $f=g \quad m_{L}$-a.e. in $\mathcal{M}\left(m_{L}\right)$, then $T(f) \stackrel{\bar{\tau}}{\approx} T(g)$; that is, if $[f]_{m_{L}}=[g]_{m_{L}}$, then $[T(f)]_{\bar{\tau}}=[T(g)]_{\bar{\tau}}$.

(3) If $T(f) \stackrel{\bar{\tau}}{\approx} T(g)$, then $f=g \quad m_{L}$-a.e.; that is, if $[T(f)]_{\bar{\tau}}=[T(g)]_{\bar{\tau}}$, then $[f]_{m_{L}}=[g]_{m_{L}}$.

Remark 5.4. Let $p$ be a given real number with $1 \leq p<+\infty$ and let $\lambda$ be a positive real number. Let $\mathcal{L}_{p}(\bar{\tau}, \lambda)$ be the space of functions $F$ of the form $F=g \circ P_{\vec{t}}$ on $C_{0}[a, b]$ where $g$ is a complex-valued function on $\mathbb{R}^{n}$ such that $F$ is in $\mathcal{M}(\bar{\tau})$ and $\left[\int_{C_{0}[a, b]}|F|^{p} d m_{\lambda}\right]^{\frac{1}{p}}=\|F\|_{\mathcal{L}_{p}(\bar{\tau}, \lambda)}$ is finite. Let $\mu_{\lambda}$ be a measure on $\mathbb{R}^{n}$ such that the Radon-Nikodým derivative $\frac{d \mu_{\lambda}}{d m_{L}}$ exists and $\frac{d \mu_{\lambda}}{d m_{L}}(\vec{u})=W_{n}(\lambda, \vec{t}, \vec{u})$. Let $\mathcal{L}_{p}\left(\mu_{\lambda}\right)$ be the space of Lebesgue measurable functions $f$ on $\mathbb{R}^{n}$ such that $\|f\|_{\mathcal{L}_{p}\left(\mu_{\lambda}\right)}=$ $\left[\int_{\mathbb{R}^{n}}\left|f\left(u_{1}, \cdots, u_{n}\right)\right|^{p} d \mu_{\lambda}\left(u_{1}, \cdots, u_{n}\right)\right]^{\frac{1}{p}}$ is finite. Let $M_{\lambda}: \mathbb{R}^{n} \rightarrow \mathbb{R}^{n}$ be a function with $M_{\lambda}(\vec{u})=\lambda \vec{u}$ (that is, $M_{\lambda}\left(u_{1}, u_{2}, \cdots, u_{n}\right)=\left(\lambda u_{1}, \lambda u_{2}, \cdots, \lambda u_{n}\right)$ ), and let $\tilde{M}_{\lambda}: C_{0}[a, b] \rightarrow C_{0}[a, b]$ be a function with $\tilde{M}_{\lambda}(x)=\lambda x$. Let $G_{\lambda}$ be an operator from $\mathcal{L}_{p}\left(\mu_{1}\right)$ into $\mathcal{L}_{p}\left(\mu_{\lambda^{-2}}\right)$ given by $G_{\lambda}(f)=f \circ M_{\lambda}$, let $H_{\lambda}$ be an operator from $\mathcal{L}_{p}(\bar{\tau}, 1)$ into $\mathcal{L}_{p}\left(\bar{\tau}, \lambda^{-1}\right)$ given by $H_{\lambda}(F)=F \circ \tilde{M}_{\lambda}$, let $T$ be an operator from $\mathcal{L}_{p}\left(\mu_{1}\right)$ into $\mathcal{L}_{p}(\bar{\tau}, 1)$ with $T(f)=f \circ P_{\vec{t}}$, and let $\tilde{T}$ be an operator from $\mathcal{L}_{p}\left(\mu_{\lambda^{-2}}\right)$ into $\mathcal{L}_{p}\left(\bar{\tau}, \lambda^{-1}\right)$ with $\tilde{T}(f)=f \circ P_{\vec{t}}$. Then, by the elementary theory of measure, we can find that the following statements hold.

(1) The operators $G_{\lambda}, H_{\lambda}, T$ and $\tilde{T}$ are all well defined, and their semi-operator norms are equal to one.

(2) $f=g \quad \mu_{1}$-a.e. if and only if $f=g \quad \mu_{\lambda}$-a.e.

(3) $f=g \quad \mu_{1}$-a.e. if and only if $T(f) \stackrel{\bar{\tau}}{\approx} T(g)$.

(4) $f=g \quad \mu_{\lambda}$-a.e. if and only if $\tilde{T}(f) \stackrel{\bar{\tau}}{\widetilde{T}}(g)$. 
(5) $H_{\lambda} \circ T=\tilde{T} \circ G_{\lambda}$; that is, the diagram

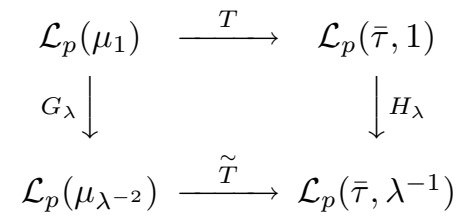

commutes.

\section{Fourier-Feynman transform and $\bar{\tau}$-MEASURABILITy}

Brue, in his Ph.D. thesis [1, written under the direction of Cameron, introduced the concept of a Fourier-Feynman transform. Since then, Fourier-Feynman transforms have been studied by Cameron, Storvick, Johnson, Skoug, Park, and many others; see [7] p. 636] for additional references. Because of measurability problems, all of the functions $F$ on $C_{0}[a, b]$ and all of the functions $f$ on $\mathbb{R}^{n}$ considered in [4], 9] were assumed to be Borel measurable; also see [8, pp. 170-171] for further discussion. In this section we will extend various results in [4], [9], in particular, Theorem 0.1, Theorem 1.1, and Theorem 2.1 of 9], to the case where the functions $F$ on $C_{0}[a, b]$ are $\bar{\tau}$-measurable and the functions $f$ on $\mathbb{R}^{n}$ are Lebesgue measurable.

Definition 6.1. Let $p$ be a given number with $1<p \leq 2$. Let $H_{n} \quad(n=1,2, \ldots)$ and $H$ be $\bar{\tau}$-measurable functions on $C_{0}[a, b]$. We say that $H$ is the $\bar{\tau}$-limit in the mean of order $p^{\prime}$ of $H_{n}$ over $C_{0}[a, b]$ provided that for each $\rho>0$,

$$
\lim _{n \rightarrow \infty} \int_{C_{0}[a, b]}\left|H_{n}(\rho y)-H(\rho y)\right|^{p^{\prime}} d \bar{m}_{1}(y)=0 .
$$

Then we write

$$
\lim _{n \rightarrow \infty}\left(W_{\tau}^{p^{\prime}}\right) H_{n} \stackrel{\bar{\tau}}{\approx} H
$$

and we call $H$ the $\bar{\tau}$-limit in the mean of order $p^{\prime}$ of $H_{n}$ over $C_{0}[a, b]$. A similar definition is understood when $n$ is replaced by a continuously varying parameter. Let $q$ be a nonzero real number. We define the $L_{p}$-analytic Fourier-Feynman transform of $F$, denoted by $\hat{T}_{q}^{(p)}(F)$, by the formula

$$
\hat{T}_{q}^{(p)}(F)(y) \equiv \lim _{\substack{\lambda \rightarrow-i q \\ \lambda \in \mathbb{C}_{+}}}\left(W_{\tau}^{p^{\prime}}\right) T_{\lambda}(F)(y)
$$

for $1<p \leq 2$ whenever this limit exists. We define the $L_{1}$-analytic FourierFeynman transform of $F$, which we denote by $\hat{T}_{q}^{(1)}(F)$, as the functional (if it exists) on $C_{0}[a, b]$ such that

$$
\hat{T}_{q}^{(1)}(F)(y) \equiv \lim _{\substack{\lambda \rightarrow-i q \\ \lambda \in \mathbb{C}_{+}}} T_{\lambda}(F)(y)
$$

for $\bar{\tau}$-a.e. $y$.

Definition 6.2. Let $n$ be a natural number and let $a=t_{0}<t_{1}<t_{2}<\cdots<t_{n} \leq b$. For $1 \leq p \leq 2$, let $\overline{\mathcal{A}}_{n}^{(p)}$ be the space of functions $F$ of the form

$$
F(x)=f\left(x\left(t_{1}\right), x\left(t_{2}\right), \cdots, x\left(t_{n}\right)\right)
$$

$\bar{\tau}$-a.e. where $f$ is an element of $L_{p}\left(\mathbb{R}^{n}\right)$. 
Let $p$ be a real number with $1 \leq p \leq 2$, and let $q$ be a nonzero real number. Let $\mathcal{M}(\bar{\tau})$ be the space of all $\bar{\tau}$-measurable functionals on $C_{0}[a, b]$ and let $\mathcal{M F}(\bar{\tau})$ be the space of all $\bar{\tau}$-measurable functionals $F$ on $C_{0}[a, b]$ such that there exists a Fourier-Feynman transform $\hat{T}_{q}^{(p)}(F)$ of $F$. Then, by the Minkowski inequality, linearity of $T_{\lambda}$ and the properties of limit, we can easily check that $\hat{T}_{q}^{(p)}$ is a linear transformation from $\mathcal{M F}(\bar{\tau})$ into $\mathcal{M}(\bar{\tau})$.

From Definitions 6.1 and 6.2, we can prove the next theorem, which is the counterpart to Theorem 0.1 in $[9]$.

Theorem 6.3. Let $q$ be a nonzero real number and let $1 \leq p \leq 2$ be given. Let $F_{1}$ and $F_{2}$ be $\bar{\tau}$-measurable on $C_{0}[a, b]$ such that $F_{1} \underset{\bar{\tau}}{\approx} F_{2}$. Then the following statements hold.

(1) $F_{1}(\cdot+y) \stackrel{\bar{\tau}}{\approx} F_{2}(\cdot+y)$ for $\bar{\tau}$-a.e. $y$.

(2) If, for $\bar{\tau}$-a.e. $y, T_{\lambda}\left(F_{1}\right)(y)$ exists for $\lambda$ in $\mathbb{C}_{+}$, then, for $\bar{\tau}$-a.e. $y, T_{\lambda}\left(F_{2}\right)(y)$ exists for $\lambda$ in $\mathbb{C}_{+}$and $T_{\lambda}\left(F_{1}\right) \stackrel{\bar{\tau}}{\approx} T_{\lambda}\left(F_{2}\right)$.

(3) If $\hat{T}_{q}^{(p)}\left(F_{1}\right)$ exists, then $\hat{T}_{q}^{(p)}\left(F_{2}\right)$ exists and $\hat{T}_{q}^{(p)}\left(F_{1}\right) \stackrel{\bar{\tau}}{\approx} \hat{T}_{q}^{(p)}\left(F_{2}\right)$.

Proof. Let $F=F_{1}-F_{2}$ and $N=\left\{x\right.$ in $\left.C_{0}[a, b] \mid F(x) \neq 0\right\}$. Then $N$ is $\bar{\tau}$ null. By Theorem 4.1, for $\bar{\tau}$-a.e. $y, N-y=\left\{x-y\right.$ in $\left.C_{0}[a, b] \mid F(x) \neq 0\right\}=\{z$ in $\left.C_{0}[a, b] \mid F(z+y) \neq 0\right\}$ is $\bar{\tau}$-null; hence we have proved statement (1). By statement (1) in this theorem, for $\bar{\tau}$-a.e. $y$ and for all positive real number $\lambda$,

$$
\int_{C_{0}[a, b]} F_{1}\left(\lambda^{-\frac{1}{2}} x+y\right) d \bar{m}_{1}(x)=\int_{C_{0}[a, b]} F_{2}\left(\lambda^{-\frac{1}{2}} x+y\right) d \bar{m}_{1}(x) .
$$

Defining $N_{1}=\left\{y\right.$ in $C_{0}[a, b] \mid T_{\lambda}\left(F_{1}\right)(y)$ does not exist for $\lambda$ in $\left.\mathbb{C}_{+}\right\}$and $N_{2}=\{y$ in $C_{0}[a, b] \mid T_{\lambda}\left(F_{1}\right)(y)$ exists but the equality (6.6) fails $\}$, we see that $N_{1} \cup N_{2}$ is $\bar{\tau}$-null and for $y$ in $\left(N_{1} \cup N_{2}\right)^{c}$ the equality (6.6) holds, which implies that for $\bar{\tau}$-a.e. $y, T_{\lambda}\left(F_{2}\right)(y)$ exists for $\lambda$ in $\mathbb{C}_{+}$and $T_{\lambda}\left(F_{1}\right) \stackrel{\bar{\tau}}{\approx} T_{\lambda}\left(F_{2}\right)$, as desired.

To prove statement (3), we will need to consider two cases: (a) $p=1$ and (b) $1<p \leq 2$.

(a) Let $N_{3}=\left\{y\right.$ in $C_{0}[a, b] \mid T_{\lambda}\left(F_{1}\right)(y) \neq T_{\lambda}\left(F_{2}\right)(y)$ for $\lambda$ in $\left.\mathbb{C}_{+}\right\}$and $N_{4}=\{y$ in $C_{0}[a, b] \mid \hat{T}_{q}^{(1)}\left(F_{1}\right)(y)$ does not exist $\}$. Then $N_{3} \cup N_{4}$ is $\bar{\tau}$-null and, for $y$ in $\left(N_{3} \cup N_{4}\right)^{c}$,

$$
\hat{T}_{q}^{(1)}\left(F_{1}\right)(y)=\lim _{\substack{\lambda \rightarrow-i q \\ \lambda \in \mathbb{C}_{+}}} T_{\lambda}\left(F_{1}\right)(y)=\lim _{\substack{\lambda \rightarrow-i q \\ \lambda \in \mathbb{C}_{+}}} T_{\lambda}\left(F_{2}\right)(y)=\hat{T}_{q}^{(1)}\left(F_{2}\right)(y),
$$

which implies that the $L_{1}$-Fourier-Feynman transform $\hat{T}_{q}^{(1)}\left(F_{2}\right)(y)$ of $F_{2}$ exists $\bar{\tau}$ a.e. $y$ and $\hat{T}_{q}^{(1)}\left(F_{1}\right) \stackrel{\bar{\tau}}{\approx} \hat{T}_{q}^{(1)}\left(F_{2}\right)$.

(b) Let $N_{5}=\left\{y\right.$ in $C_{0}[a, b] \mid \hat{T}_{q}^{(p)}\left(F_{1}\right)(y)$ is not well-defined $\}$. Then $N_{3} \cup N_{5}$ is $\bar{\tau}$-null. Letting $\hat{T}_{q}^{(p)}\left(F_{2}\right)(y)=\hat{T}_{q}^{(p)}\left(F_{1}\right)(y)$ on $\left(N_{3} \cup N_{5}\right)^{c}$ and $\hat{T}_{q}^{(p)}\left(F_{2}\right)(y)=0$ on 
$N_{3} \cup N_{5}$, we get $\hat{T}_{q}^{(p)}\left(F_{1}\right) \stackrel{\bar{\tau}}{\approx} \hat{T}_{q}^{(p)}\left(F_{2}\right)$ and, for all positive real numbers $\rho$,

$$
\begin{aligned}
& \lim _{\substack{\lambda \rightarrow-i q \\
\lambda \in \mathbb{C}_{+}}} \int_{C_{0}[a, b]}\left|\left(T_{\lambda}\left(F_{2}\right)-\hat{T}_{q}^{(p)}\left(F_{2}\right)\right)(\rho y)\right|^{p^{\prime}} d \bar{m}_{1}(y) \\
& \quad=\lim _{\substack{\lambda \rightarrow-i q \\
\lambda \in \mathbb{C}_{+}}} \int_{C_{0}[a, b] \backslash\left(N_{3} \cup N_{5}\right)}\left|T_{\lambda}\left(F_{2}\right)(\rho y)-\hat{T}_{q}^{(p)}\left(F_{2}\right)(\rho y)\right|^{p^{\prime}} d \bar{m}_{1}(y) \\
& \quad=\lim _{\substack{\lambda \rightarrow-i q \\
\lambda \in \mathbb{C}_{+}}} \int_{C_{0}[a, b] \backslash\left(N_{3} \cup N_{5}\right)}\left|T_{\lambda}\left(F_{1}\right)(\rho y)-\hat{T}_{q}^{(p)}\left(F_{2}\right)(\rho y)\right|^{p^{\prime}} d \bar{m}_{1}(y) \\
& \quad=\lim _{\substack{\lambda \rightarrow-i q \\
\lambda \in \mathbb{C}_{+}}} \int_{C_{0}[a, b]}\left|T_{\lambda}\left(F_{1}\right)(\rho y)-\hat{T}_{q}^{(p)}\left(F_{2}\right)(\rho y)\right|^{p^{\prime}} d \bar{m}_{1}(y) \\
& =0 .
\end{aligned}
$$

Hence, we have

$$
\hat{T}_{q}^{(p)}\left(F_{2}\right) \stackrel{\bar{\tau}}{\approx} \lim _{\substack{\lambda \rightarrow-i q \\ \lambda \in \mathbb{C}_{+}}}\left(W_{\tau}^{p^{\prime}}\right) T_{\lambda}\left(F_{2}\right)
$$

as desired.

Using Theorem 6.3, we can improve Theorems 1.1 and 1.2 in [9] as follows.

Theorem 6.4. Let $1 \leq p \leq 2$ and let $q$ be a non-zero real number. Let $F$ in $\overline{\mathcal{A}}_{n}^{(p)}$ be given by (6.5). Then:

(1) The $L_{p}$-analytic Fourier-Feynman transform of $F$, namely $\hat{T}_{q}^{(p)}(F)$, exists, is in $\overline{\mathcal{A}}_{n}^{(p)}$ and is given by the formula

$$
\hat{T}_{q}^{(p)}(F) \stackrel{\bar{\tau}}{\approx}\left(K_{-i q} f\right)
$$

(2) For each $\rho>0$,

$$
\lim _{\substack{\lambda \rightarrow-i q \\ \lambda \in \mathbb{C}_{+}}} \int_{C_{0}[a, b]}\left|T_{\bar{\lambda}} T_{\lambda}(F)(\rho y)-F(\rho y)\right|^{p} d \bar{m}_{1}(y)=0 .
$$

(3) $T_{\bar{\lambda}} T_{\lambda}(F) \rightarrow F \quad \bar{\tau}$-a.e. as $\lambda \rightarrow-i q$ through $\mathbb{C}_{+}$.

Proof. Using the above Theorem 5.2 and Lemmas 1.1-1.3 in [9], we can prove statements (1) and (2) by the same method as in the proofs of Lemmas 1.1-1.3 in 9 if we replace Borel measurability by Lebesgue measurability and the concept of s-a.e. by the concept of $\bar{\tau}$-a.e. By statement (1) in this theorem, we know that $T_{\lambda}(F)$ and $T_{\bar{\lambda}} T_{\lambda}(F)$ are in $\overline{\mathcal{A}}_{n}^{(p)}$ for $\lambda$ in $\mathbb{C}_{+}$, and by Theorem 5.2, $T_{\lambda}(F)$ and $T_{\bar{\lambda}} T_{\lambda}(F)$ are all $\bar{\tau}$-measurable. Hence, putting $N=\left\{y\right.$ in $C_{0}[a, b] \mid$ the limit $\lim _{\substack{\lambda \rightarrow-i q \\ \lambda \in \mathbb{C}_{+}}} T_{\bar{\lambda}} T_{\lambda}(F)(y)$ does not converge to $\left.F(y)\right\}$, we see that $N$ is $\bar{\tau}$-measurable. By statement (3) in Lemma 1.3 in [9], we know that $\rho N$ is $\bar{m}_{1}$-null for all positive real numbers $\rho$. Therefore, $N$ is $\bar{\tau}$-null, as desired.

\section{ACKnowledgement}

The authors would like to express their sincere gratitude to Professor M. L. Lapidus of the University of California, Riverside, for a number of valuable comments during the preparation of this paper. 


\section{REFERENCES}

1. M. D. Brue, A functional transform for Feynman integral similar to the Fourier transform, Ph. D. Dissertation, U. Minnesota (1972).

2. R. H. Cameron, The translation pathology of Wiener space, Duke Math. J., 21 (1954), 623-627. MR 16:375b

3. R. H. Cameron and W. T. Martin, The behavior of measure and measurability under change of scale in Wiener space, Bull. Amer. Math. Soc., Vol. 53 (1947), 130-137. MR 8:392a

4. R. H. Cameron and D. A. Storvick, An $L_{2}$-analytic Fourier-Feynman transform, Michigan Math. J., 23 (1976), 1-30. MR 53:8371

5. K. S. Chang and K. S. Ryu, A generalized converse measurability theorem, Proceedings of the American Mathematical Society, Vol. 104, No. 3 (1988), 835-839. MR 89e:28021

6. E. Hewitt and K. Stromberg, Real and abstract analysis. A modern treatment of the theory of functions of a real variable, Springer-Verlag, New York, (1965). MR 32:5826

7. G. W. Johnson and M. L. Lapidus, The Feynman integral and Feynman's operational calculus, Oxford Mathematical Monographs, Clarendon Press, Oxford (2000). MR 2001i:58015

8. G. W. Johnson and D. L. Skoug, Scale-invariant measurability in Wiener space, Pacific J. Math., Vol. 83, No. 1 (1979), 157-176. MR 81b:28016

9. G. W. Johnson and D. L. Skoug, An $L_{p}$-analytic Fourier-Feynman transform, Michigan Math. J., 26 (1979), 103-127. MR 81a:46050

10. E. J. McShane, Families of measures and representations of algebras of operators, Trans. Amer. Math. Soc. 102 (1962), 328-345. MR 25:462

11. M. M. Rao, Measure theory and integration, Pure and Applied Mathematics, John Wiley and Sons Inc., New York (1987). MR 89k:28001

12. H. L. Royden, Real analysis, Third edition, Macmillan Publishing Company, New York (1988). MR 90g:00004

13. K. S. Ryu, A property of Borel subsets of Wiener space, J. Chungcheng Math. Soc., Vol. 14 (1991), 45-48.

14. N. Wiener, Differential space, J. Math. Phys., 58 (1923), 131-174.

15. Y. Yamasaki, Measures on infinite-dimensional spaces, World Scientific Series in Pure Mathematics, Vol. 15 (1985). MR 90b:28015

16. J. Yeh, Stochastic process and the Wiener integral, Pure and Applied Mathematics, Vol. 13, Marcel Dekker, Inc., New York (1973). MR 57:14166

Department of Mathematics, Han Nam University, Taejon 306-791, Korea

E-mail address: ksr@math.hannam.ac.kr

Department of Mathematics, Han Nam University, Taejon 306-791, Korea

E-mail address: mki@mail.hannam.ac.kr 